\section{Evaluación geriátrica integral del adulto mayor}

\author{
ALMA ROSA CORTÉS N. ${ }^{1,2}$, ENRIQUE VILLARREAL R. ${ }^{1}$, \\ LILIANA GALICIA R. ${ }^{1, \mathrm{a}}$, LIDIA MARTÍNEZ G. ${ }^{1, \mathrm{a}}$, EMMA ROSA VARGAS D. ${ }^{1, \mathrm{~b}}$
}

\section{Cross sectional geriatric assessment of Mexican older people}

Background: Due to the progressive aging of our population, it is imperative to evaluate the life conditions and health limitations of older people. Aim: To report the results of an integral geriatric evaluation of Mexican older people. Material and Methods: A cross-sectional assessment of 324 older subjects of a median age of 70 years (57\% females) beneficiaries of the Mexican Institute of Social Security of the city of Querétaro. Social, demographic, medical, functional and cognitive variables were evaluated. Results: Of the studied subjects, 37\% were illiterate, $61 \%$ lived with a partner and $47 \%$ were dedicated to household activities. Thirty three percent had visual impairment, 54\% had hearing impairment, 39\% had urinary incontinence, $26 \%$ reported falls in the last six months, 34\% had nutritional problems, 38\% were functionally dependent, $49 \%$ had sleeping problems, $25 \%$ had cognitive impairment and 25\% had depression. Conclusions: The general health outlook of this population is encouraging, considering that more than half are not functionally impaired.

(Rev Med Chile 2011; 139: 725-731).

Key words: Aged; Geriatric assessment; Mexico.

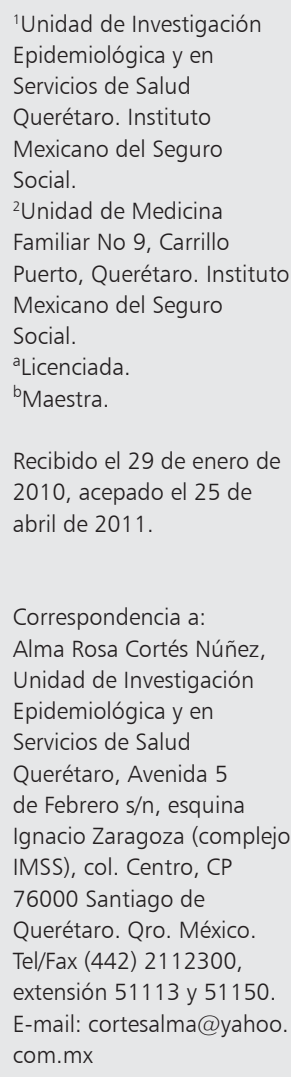

E l envejecimiento poblacional, producto de la transición demográfica y epidemiológica y la consecuente elevación en la frecuencia y número de enfermedades crónico degenerativas causantes de invalidez, plantea retos importantes al Sistema de Salud por la gran demanda de servicios especializados, pues tendrá que atender a un número creciente de población adulta mayor con limitados recursos y con un sistema tradicional aún no acondicionado a un alto costo ${ }^{1}$.

El deterioro que va ocurriendo asociado al proceso de envejecimiento ha dado lugar a la creencia errónea de que éste en sí mismo corresponde a una patología, la situación de salud del adulto mayor, imagen incompleta considerando que se conforma por datos inevitablemente de mortalidad, en los que obviamente no se reflejan los problemas que no conducen a la muerte, los de morbilidad que se expresan por la demanda de consulta. Por otra parte, estos señalan la patología que el médico o el paciente mismo consideran como principal motivo de consulta, dejando pasar entidades patológicas particulares de mayor frecuencia en esta etapa de la vida ${ }^{2}$.

La evaluación geriátrica integral (EGI) es un proceso diagnóstico multidimencional e interdisciplinario, diseñado para identificar y cuantificar los problemas físicos, funcionales, psíquicos y sociales que provocan alteraciones que con frecuencia llevan al adulto mayor a la incapacidad como deterioro cognitivo/demencia ${ }^{3}$, trastornos de ánimo/depresión ${ }^{4}$, trastornos del sueño/insomnio $^{5}$, inmovilidad/encamamiento $0^{6}$, inestabilidad/ caídas $^{7}$, incontinencia urinaria ${ }^{8}$, deprivación sensorial (vista y audición) ${ }^{9,10}$, malnutrición ${ }^{11,12-14}$, iatrogenia/fármaco y sociales/ sistemas de apoyo ${ }^{15}$. El objetivo es desarrollar un plan de tratamiento y seguimiento de dichos problemas, con el fin 
de disminuir la morbilidad y la mortalidad de la población geriátrica y mejorar su calidad de vida, así como la óptima utilización de recursos para afrontarlos.

En la literatura se mencionan reportes de estudios de EGI en poblaciones diferentes de adultos mayores ${ }^{15-17}$, utilizando diversas escalas u omitiendo algunos problemas de salud ${ }^{18-20}$, estos trabajos publicados han demostrado mejorar al menos uno de estos aspectos y otros muestran grandes beneficios $^{21}$ por lo tanto, llevar a cabo como tal la EGI nos llevará a identificar los problemas potenciales de salud y permitirá enfocar, dirigir y realizar acciones en beneficio de esta población.

La EGI constituye la forma más razonable de aproximarse al adulto mayor desde cualquier nivel de atención y esencial su aplicación para mejorar su calidad de vida. Los adultos mayores frágiles corren peligro de perder aquello que más estiman en el ámbito de la salud, su autonomía personal. Con el paso de los años ha cobrado importancia debido a que permite realizar una evaluación precisa y adecuada de los distintos aspectos que condicionan la salud de los adultos mayores. Esta intervención demuestra un impacto más sobre la percepción individual de salud, menor número de visitas a los servicios de consulta, mayor desempeño en las actividades instrumentales de la vida diaria, mejoría en su vida social y del estado de ánimo y mental. El modelo tradicional de aproximación clínica ha demostrado que la aplicación de este modelo al adulto mayor, especialmente al "adulto mayor frágil" tiene como consecuencia una mayor frecuencia de diagnóstico incompletos, una excesiva prescripción de fármacos, una mayor incapacidad funcional, al utilizar con menos frecuencia la rehabilitación, una utilización inapropiada de los recursos sociales y una institucionalización menos adecuada del paciente ${ }^{22,23}$.

Así de esta forma, frente a un modelo clásico de aproximación biomédica al adulto mayor, los múltiples e interrelacionados problemas del paciente geriátrico requieren de una aproximación biopsicológica, social y funcional con el fin de alcanzar un plan de tratamiento global, optimizar la utilización de los recursos y garantizar el seguimiento de los casos.

Por lo que en este trabajo se planteó identificar el panorama de salud del adulto mayor a través de la evaluación geriátrica integral en la zona metropolitana de la ciudad de Querétaro.

\section{Metodología}

Se realizó un estudio transversal descriptivo, con adultos mayores de 60 años y más, usuarios ambulatorios de ambos sexos, derechohabientes del Instituto Mexicano del Seguro Social (IMSS) que acudieron al primer nivel de atención de las siete Unidades de Medicina Familiar (UMF) de la zona metropolitana de la ciudad de Querétaro de febrero a diciembre de 2007. Se incluyeron a todos aquellos que asistieron a consulta y que aceptaron participar con previo consentimiento informado. El tamaño de muestra se obtuvo a través de la fórmula para población infinita $(\mathrm{z}=1,64, \mathrm{p}=0,3, \mathrm{q}=$ $0,07, \mathrm{~d}=0,05)$, tomando la hipótesis estado nutricio para el mayor tamaño de la muestra $(\mathrm{n}=324)$. La técnica muestral fue ponderada por unidad de adscripción, distribuyéndose proporcionalmente de acuerdo a la población derechohabiente de cada unidad, y por consultorio. La selección al interior fue por cuota. La encuesta se realizó por el investigador, durante el tiempo de espera de la consulta o posterior a ésta.

Se analizaron variables sociodemográficas (edad, sexo, escolaridad y ocupación). La evaluación geriátrica integral incluyó variables clínicomédicas (alteraciones de visión, audición, incontinencia urinaria, malnutrición, inestabilidad y caídas), funcionales físicas (actividades de la vida diaria) y de capacidad mental (deterioro cognitivo, depresión, trastornos del sueño).

\section{Variables clínico-médicas}

Visión. Se le preguntó al adulto mayor si tenía problemas para ver la televisión, leer o para ejecutar cualquier actividad de la vida diaria (percepción) y se procedió a evaluar la agudeza visual mediante la carta de Snellen (con o sin lentes) se le pidió colocarse a una distancia de tres metros, explorando cada ojo por separado, tomando como punto de corte la agudeza visual de 20/40 .

Audición. Se preguntó si tenía dificultad para oír (percepción) y se procedió a aplicar, el método del susurro. El examinador se puso de pie por detrás del paciente y fuera de su campo visual, a $60 \mathrm{~cm}$ de distancia (examinando cada oído por separado). Se hizo presión con un dedo en el oído no explorado, se susurraron tres números y letras combinados para posteriormente solicitar al paciente repitiera lo susurrado. En caso de un error, se repitió la prueba con otros números y letras dife- 
rentes. Se consideró sin alteraciones, si el paciente repitió al menos tres de los seis números y letras. Posteriormente se exploró el oído contrario ${ }^{10}$.

Incontinencia urinaria. Se preguntó si en el último año tuvo pérdidas involuntarias de orina, con respuesta afirmativa se consideró como problema ${ }^{11}$.

Caídas y dificultad para caminar. Se preguntó si en el último año sufrió caída o si tenía inseguridad y dificultad para caminar, de ser afirmativa se procedió a realizar la prueba "Levántate y anda" (prueba de marcha). Se le pidió al adulto mayor sentado en una silla que se levantara y permaneciera momentáneamente de pie, caminara en línea recta aproximadamente tres metros, diera la vuelta sin tocar la pared, regresara nuevamente al mismo lugar y se sentara de nuevo. Se evaluó la velocidad de la marcha, la longitud del paso, la base de sustentación, la regularidad del paso y la relación entre el tiempo de apoyo de ambos pies y uno solo. Los adultos mayores capaces de completar las indicaciones en menos de 20 segundos se consideraron sin problemas de marcha ${ }^{7}$.

Estado nutricional. Se evaluó mediante el cálculo del índice de masa corporal (IMC), (IMC = peso en kilogramos/estatura en metros elevado al cuadrado). En los adultos mayores el punto de corte para definir el peso normal fue de 22,0-29,9, para desnutrición un IMC menor de 18,5, como obesidad igual o mayor a 30,0, y como riesgo de desnutrición un IMC entre 18,5 y 21,9 $9^{11-13}$.

\section{Variables funcionales físicas}

Se le preguntó sobre sus actividades de la vida diaria. Se midió a través del índice de Barthel, con puntos de corte 0-20 dependencia total, 21-60 dependencia severa, 61-90 dependencia moderada, 91-99 dependencia escasa y 100 independencia ${ }^{6}$.

\section{Variables de capacidad mental}

Deterioro cognitivo. A través de la escala del estado mental de Folstein, se valoró orientación, memoria reciente y diferida, así como lenguaje. Los niveles de deterioro cognitivo tuvieron los siguientes puntos de corte de 30-25 normal, 2420 deterioro leve, 19-16 deterioro moderado, 15 y menos deterioro grave ${ }^{3}$.

Depresión. Se aplicó la escala de geriátrica de Yesavage, que mide la percepción del sujeto sobre cómo se sintió en la última semana, con respuestas de si/no. Los niveles de depresión tuvieron los si- guientes puntos de corte $0-5$ normal, 6-9 probable depresión, 10 y más depresión establecida ${ }^{4}$.

Trastornos del sueño. Se evaluó el último mes a través de tres preguntas, 1) para conciliar el sueño ¿tarda más de 30 min?; 2) por la noche ¿duerme menos de 6 hrs?; 3 ) al levantarse por la mañana ¿se siente cansado? al contestar en forma afirmativa al menos una se consideró trastornos del sueño ${ }^{5}$.

El plan de análisis incluyó promedios, porcentajes e intervalos de confianza.

\section{Resultados}

El promedio de edad de la población fue 70,40 años (IC 95\%; 69,59-71,21), predominó el género femenino 57,1\% (IC 95\%; 51,7-62,5), con pareja $61,1 \%$ (IC 95\%; 55,8-66,4), analfabetas 37,0\% (IC $95 \% ; 31,7-42,3)$ y $47,5 \%$ (IC 95\%; 42,1-52,9) se dedicaba al hogar.

\section{Problemas clínico-médicos}

El 59,0\% (IC 95\%; 53,6-64,4) percibía tener problemas para ver la televisión, leer o para ejecutar cualquier actividad de la vida diaria, $y$ $33,3 \%$ (IC 95\%; 28,2-38,4) presentaba una medición de agudeza visual de ojo derecho igual o mayor de 20/50, 43,2\% (IC 95\%; 37,8-48,6) percibía problemas para oír, con predominio en el oído derecho 54,0\% (IC 95\%; 48,6-59,4) (Tabla 1).

El 38,6\% (IC 95\%; 33,3-43,9) manifiestó tener pérdida involuntaria de orina en el último año. El 51,1\% (IC 95\%; 45,7-56,6) refirió problemas de caídas y dificultad para caminar en el último año, 34,0\% (IC 95\%; 28,8-39,2) presentó problemas nutricios. Resto de información se aprecia en la Tabla 1.

\section{Funcionalidad física}

El 38,2\% (IC 95\%; 32,9-43,5) tenía algún grado de dependencia para realizar sus actividades de la vida diaria (Tabla 2).

\section{Capacidad mental}

El 24,7\% (IC 95\%; 0,0-29,4) de la población presentaba algún grado de deterioro cognitivo, así como 25\% (IC 95\%; 0,0-29,7) depresión; 49,4\% (IC 95\%; 44,0-54,8) manifiestó tener problemas para conciliar el sueño en el último mes, 41\% (IC 95\%; 41,0-46,4) dormía menos de 6 horas y cerca de la mitad al levantarse por la mañana se sentía cansado, (Tabla 3). 
Tabla 1. Características clínico médicas de la población adulto mayor

\begin{tabular}{|c|c|c|c|c|}
\hline \multirow[t]{2}{*}{ Características } & & \multirow[t]{2}{*}{$\%$} & \multicolumn{2}{|c|}{ IC $95 \%$} \\
\hline & & & Mín. & Máx. \\
\hline \multicolumn{5}{|l|}{ Visión } \\
\hline \multirow{2}{*}{\multicolumn{5}{|c|}{$\begin{array}{l}\text { Percepción de problemas para ver la televisión, leer o } \\
\text { para ejecutar cualquier actividad de la vida diaria }\end{array}$}} \\
\hline & & & & \\
\hline \multicolumn{2}{|l|}{ Alteración de la visión (20/50 y más) } & 33,3 & 28,2 & 38,4 \\
\hline \multicolumn{5}{|l|}{ Alteración ojo izquierdo } \\
\hline \multicolumn{2}{|l|}{ Alteración de la visión (20/50 y más) } & 31,8 & 26,7 & 36,9 \\
\hline \multicolumn{5}{|l|}{ Audición } \\
\hline Percepción de tener dificultad para oír & Sí & 43,2 & 37,8 & 48,6 \\
\hline Alteración auditiva derecho & Sí & 54,0 & 48,6 & 59,4 \\
\hline Alteración auditiva izquierdo & Sí & 49,1 & 43,7 & 54,5 \\
\hline \multicolumn{5}{|l|}{ Incontinencia urinaria } \\
\hline Incontinencia urinaria en el último año & Sí & 38,6 & 33,3 & 43,9 \\
\hline \multicolumn{5}{|l|}{ Caídas } \\
\hline Caídas en el último año y dificultad para caminar & Sí & 51,1 & 45,7 & 56,6 \\
\hline Dificultad para caminar & Sí & 50,3 & 44,9 & 55,7 \\
\hline \multicolumn{5}{|l|}{ Estado nutricio } \\
\hline \multicolumn{5}{|l|}{ Índice de masa corporal } \\
\hline Desnutrición & & 0,6 & 0,0 & 1,4 \\
\hline Riesgo desnutrición & & 6,2 & 3,6 & 8,8 \\
\hline Normal & & 66,0 & 60,8 & 71,2 \\
\hline Obesidad & & 27,2 & 22,4 & 32,0 \\
\hline
\end{tabular}

Tabla 2. Características de funcionalidad física de la población adulto mayor

\begin{tabular}{|lccc|}
\hline Características & $\%$ & \multicolumn{2}{c|}{ IC $\mathbf{9 5 \%}$} \\
\hline Grado de dependencia física & & & \\
\hline Dependencia total & 0,6 & 0,0 & 1,4 \\
\hline Dependencia severa & 1,5 & 0,2 & 2,8 \\
\hline Dependencia moderada & 15,4 & 11,5 & 19,3 \\
\hline Dependencia escasa & 20,7 & 16,3 & 25,1 \\
\hline Independencia & 61,7 & 56,4 & 67,0 \\
\hline
\end{tabular}

\section{Discusión}

La valoración integral del adulto mayor como método diagnóstico permite establecer un panorama general de salud de esta población.

En la literatura se encuentran diversos estudios de EGI, incluyendo adultos mayores con edad de 60 y más años, ambos sexos, ambulatorios, usando una serie de escalas apropiadas para cada evento, siendo los resultados similares a los encontrados en el presente estudio y todos confluyen en el punto de la importancia de su aplicación ${ }^{6-21}$.

En el proceso de envejecimiento se presenta una serie de eventos que de no ser identificados en forma oportuna tienen efectos devastadores en la calidad de vida del adulto mayor. 
Evaluación geriátrica integral - A. R. Cortés et al

Tabla 3. Características de trastornos del sueño y capacidad mental de la población de adulto mayor

\begin{tabular}{|c|c|c|c|c|}
\hline \multirow[t]{2}{*}{ Características } & & \multirow[t]{2}{*}{$\%$} & \multicolumn{2}{|c|}{ IC $95 \%$} \\
\hline & & & Mín. & Máx. \\
\hline \multicolumn{5}{|l|}{ Deterioro cognitivo } \\
\hline Deterioro severo & & 0,6 & 0,0 & 1,4 \\
\hline Deterioro moderado & & 3,1 & 1,2 & 5,0 \\
\hline Deterioro leve & & 21,0 & 16,6 & 25,4 \\
\hline Sin deterioro & & 75,3 & 70,6 & 80,0 \\
\hline \multicolumn{5}{|l|}{ Depresión } \\
\hline Sin depresión & & 75,0 & 70,3 & 79,7 \\
\hline Probable & & 21,9 & 17,4 & 26,4 \\
\hline Establecida & & 3,1 & 1,2 & 5,0 \\
\hline \multicolumn{5}{|l|}{ Trastornos del sueño } \\
\hline Para conciliar el sueño ¿tarda más de 30 min? & Sí & 49,4 & 44,0 & 54,8 \\
\hline Por la noche ¿duerme menos de 6 hrs? & Sí & 41,0 & 35,6 & 46,4 \\
\hline Al levantarse por la mañana ¿se siente cansado? & Sí & 45,4 & 40,0 & 50,8 \\
\hline
\end{tabular}

La disminución de la visión y de la audición ocupan el segundo lugar en prevalencia tras los problemas osteoarticulares. Dentro de los cambios en el proceso de envejecimiento la dificultad de acomodación del cristalino para enfocar objetos cercanos, la presbicia, es frecuente en este grupo de edad, encontrándose presente en un tercio de la población.

Las alteración de cualquier componente del sistema auditivo con disminución de la percepción de las frecuencias altas originan diferentes grados de sordera, hallándose en más de la mitad de los adultos mayores estudiados. Dichas alteraciones tienen amplias repercusiones en el ámbito funcional, psicológico y social, siendo un elemento de primer orden en cuanto a riesgo de caídas y accidentes, aunado a la incontinencia urinaria, presente en un tercio de la población, no como causa directa del envejecimiento pero sí determinada por una serie de cambios anatómicos y funcionales que pueden influir en menor o mayor grado para que se presente, incrementando el aislamiento, sentimiento de humillación personal, vergüenza, inseguridad, ansiedad, depresión y tristeza, caídas, dependencia, predisposición a la institucionalización, infecciones, trastornos nutricios llevando al adulto mayor a la desnutrición, con un estado inmunológico ya comprometido haciéndolo más susceptible a infecciones y complicaciones.

Se encontró discordancia en la prevalencia de desnutrición y riesgo de desnutrición, siendo más baja en relación a los reportado con otros autores, no así con la obesidad. Es posible que estos resultados tengan cierto sesgo por cambios artificiales en el adulto mayor como por ejemplo una mayor retención de agua, edemas, aumentando su peso corporal y así su IMC.

Si bien la causa de caídas y dificultad de la marcha es multifactorial, encontrándose presente en la mitad de esta población, se han identificado factores de riesgo condicionantes como los ya mencionados con repercusiones que van desde lesiones físicas, fracturas, vergüenza, mayor aislamiento social, síndrome post caída, hospitalización, llegando en algunos casos hasta la muerte. Toda esta cascada de eventos aunada a trastornos en el sueño presente en la mitad de los adultos estudiados repercute en la calidad de vida, acentuando la depresión, padecimiento psicogeriátrico más frecuentes en este grupo de edad, ya que en esta etapa de la vida todo es pérdidas.

La depresión frecuentemente no es identificada en etapas tempranas, presente en una cuarta parte al igual que el estado cognitivo que debido entre 
otras cambios a la atrofia cerebral y engrosamiento de las meninges hay disminución de las respuestas intelectuales y capacidad de razonamiento, así como disminución en el análisis e integración de la información sensorial, provocando disminución en la memoria a corto plazo y alguna pérdida en la habilidad de aprendizaje, aunque este no es un padecimiento exclusivo de la vejez, la edad por sí misma es un factor de riesgo para padecerla, su prevalencia se duplica cada 5 años después de los 65 años, en los mayores de 85 años de edad alcanza una prevalencia de 20 al 50\%.

La Organización Panamericana de la Salud (OPS) enfatiza que la salud del adulto mayor debe medirse en términos de conservación de la funciona y no en razón de lo que se ha perdido, por lo tanto en este estudio se encontró un mayor porcentaje de independencia para las actividades de la vida diaria, ya que más de la mitad son autosuficientes, esto es explicable ya que la población estudiada es una población que se puede considerar joven y que asistían a consulta de medicina familiar. Por todo lo anterior, se puede concluir que el panorama de salud de la población estudiada es alentador por tratarse de una población de adulto mayor joven, en la cual más de la mitad tiene autonomía para las actividades de la vida diaria. Estos resultados marcan una gran área de oportunidad, esto es de relevancia ya que a través de la EGI en el primer nivel de atención se pueda incidir en la prevención e identificación de problemas de salud del adulto mayor para adecuado manejo y que éste llegue a viejo-viejo en las mejores condiciones y una mejor calidad de vida.

\section{Referencias}

1. Villanueva EA. Sobre el envejecimiento: una perspectiva integral. Rev Hosp Gral Dr M Gea González 2000; 3 (3): 107-14.

2. García GJJ. Perfil epidemiológico en el adulto mayor. Rev. Fac Med UNAM, 2000.

3. Beaman S, Beaman P, García-Peña C, Villa M, Heres J, Cordova A, et al. Validation of a modified version of the minimental state examination (MMSE) in Spanish. Aging Neuropsychology and Cognition 2004; 11 (1): $1-11$.

4. Belló M, Puentes-Rosas E, Medina-Mora M, Lozano R. Prevalencia y diagnóstico de depresión en población adulta en México. Salud Publica Mex 2005; 47 (S1): S4S11.
5. Harvey AG. Insomnia: symptom or diagnosis? Clin Psychol Rev 2001; 21: 1037-59.

6. Hsueh IP, Lee MM, Hsieh CL. The psychometric characteristics of the Barthel activities of daily living index in stroke patients. J Formos Med Assoc 2001; 100: 526-32.

7. Podsiadlo D, Richardson S. The timed "Up and go": a test of basic functional mobility for frail elderly persons. J Am Geriatr Soc 1991; 39: 142-8.

8. International Continence Society. Standardization of terminology of lower urinary tract function. Scand J Urol Nephrol 1988 Suppl; 114: 5-19.

9. Schneck ME, Haegerstrom-Portnoy G. Practical assessment of vision in the elderly. Ophthalmol Clin North Am 2003; 16 (2): 269-87.

10. Bagai A, Thavendiranathan P, Detsky AS. Does this patient have hearing impairment? JAMA 2006; 295: 416-28.

11. Douketis JD, Paradis G, Keller H, Martineau C. Canadian guidelines for body weight classification in adults: application in clinical practice to screen for overweight and obesity and to assess disease risk. CMAJ 2005; 172 (8): 995-8.

12. Nutrition support for adults. Oral nutrition support, enteral tube feeding and paranteral nutrition. National Collaborating Centre for Acute Care. London, 2006. Disponible en: www.rcsens.acuk

13. Vellas B, Guigoz Y, Baumgartner R, Garry P, Lauque S, Albarede JL. Relationships between nutricional markers and the mini-nutritional assessment in 155 older persons. J Am Geriatr Soc 2000; 48: 1300-9.

14. García-Navarro JA. Niveles de atención geriátrica. En: Rivera CJM, Cruz JAJ. Geriatría en atención primaria. $3^{\mathrm{a}}$ edición. Barcelona. Aula Médica, 2002. pp. 135-46.

15. Villanueva V. Evaluación del paciente anciano. Revista de postgrado de la cátedra vía medicina-UNNE.2000. [acceso 22 noviembre 2008] Disponible en: http://med. unne.edu.ar/revista 98/evaluación_paciente_anciano. htm

16. Villar ND, Varela PL, Chigne O. Evaluación integral en pacientes hospitalizados y ambulatorios. Boletín de la Sociedad Peruana de Medicina Interna 2000; 13 (3).

17. Lisigurski-Teitelman M, Varela-Pinedo L, Ortiz-Saavedra JP. Valoración geriátrica integral en una población de adultos mayores. Revista de la Sociedad Peruana. (spmi/ Vol15_N1/encabezado.htm)

18. Salinas-Martínez R, Vázquez-García A, Pérez-Chávez F, Garza-Cantú M, Barragán-Berlanga AJ. Evaluación geriátrica de la población de jubilados de la Universidad Autónoma de Nuevo León. Revistas Médicas Mexicanas, 2003; 5 (21): 221-7. Disponible en: www.revistamedicasmexicanas.com.mx 
19. Reyes-Frausto S. Population ageing in the Mexican institute of social security: health policy and economic implications. Primera edición. México. Unidad de Investigación Médica sobre el Envejecimiento 2001; pp. $1-252$.

20. Díaz TV, Díaz TI, Rojas CG, Novogrodsky RD. Evaluación geriátrica en la atención primaria. Rev Med Chile 2003; 131: 895-901.

21. My GE, Ramos L, Libre Rodríguez MJ, Samper NJ, Prado
TL. Propuesta de evaluación geriátrica para su aplicación en los centros asistenciales de las Fuerzas Armadas Revolucionarias. Rev Cubana Milit 2005; 34 (1).

22. Gamarra P, Gómez M. La necesidad de la evaluación geriátrica integral. Boletín de la Sociedad Peruana de Medicina Interna 1997; 10: 143-52.

23. Ribera-Casado J. Geriatría: conceptos y generalidades. Medicina Interna de Farreras. España, Mosby Edits; 1997; pp. 1275-300. 